\title{
CONVERGENT HIGHER DERIVATIONS ON LOCAL RINGS
}

\author{
BY \\ NICKOLAS HEEREMA( $\left.{ }^{1}\right)$
}

I. Introduction. In this paper we define a quasi-local ring $R$, or $(R, M)$, to be a commutative ring with unity having a unique maximal ideal $M$ such that $\bigcap_{n=1}^{\infty} M^{n}=\{0\}$. Thus a Noetherian quasi-local ring is a local ring. A higher derivation $D=\left\{D_{i}\right\}_{i=1}^{\infty}$ on a quasi-local ring $R$ is said to be convergent if, for all $a$ in $R, \sum_{i=0}^{\infty} D_{i}(a)$ is a convergent series in the $M$-adic topology. $D_{0}$ always denotes the identity mapping. If $R$ is complete the mapping $\alpha_{D}: a \rightarrow \sum_{i=0}^{\infty} D_{i}(a)$ is an endomorphism of $R$ which induces the identity mapping on the residue field of $R$ (Lemma 1). With suitable restrictions on $D, \alpha_{D}$ is an automorphism and hence an inertial automorphism. A seemingly "natural" additional condition sufficient to insure that $\alpha_{D}$ is an automorphism is the condition

$$
D_{i}(M) \subset M^{2}, \quad i \geqq 1 .
$$

A convergent higher derivation which satisfies (1) is said to be $M$-convergent.

In a number of recent papers [4], [5], [7], Neggers, Wishart, and the author have used convergent higher derivations to study the inertial automorphisms of particular kinds of complete local rings. In particular Neggers [5] used higher derivations to relate properties of the higher ramification groups of a ramified $v$-ring to its derivation structure. The author has shown [4, Theorem 3.1] that if $R$ is an unramified $n$-dimensional complete regular local ring then every inertial automorphism of $R$ is of the form $\alpha_{D}$ where $D=\left\{D_{i_{1}}, \ldots, i_{n}\right\}$ is a convergent higher derivation on $n$-indices. By defining $H_{m}$ to be $\sum_{i_{1}+\cdots+i_{n}=m} D_{i_{1}} \ldots \ldots i_{n}$ one obtains a higher derivation $H$ on one index such that $\alpha_{H}=\alpha_{D}$, and $H$ is, in fact, what is called "strongly convergent" in this paper (Definition 3). The representation of inertial automorphisms by higher derivations provides a convenient means for determining the factor groups of the higher ramification groups of $R$ in this case $[4$, Theorems 2.1, 2.2, 2.3].

This paper is primarily concerned with convergent higher derivations as such. A bit of calculation with the possibility of defining a composition of higher derivations so that the condition $\alpha_{D \circ D^{\prime}}=\alpha_{D} \alpha_{D^{\prime}}$ obtains leads to Definition 2. Theorem 1 asserts that the set of all higher derivations $H(R, R)$ on any (noncommutative) ring $R$ is a group with respect to this composition. §II is concerned with closure properties of various convergent subsets of $H(R, R)$ with respect to both the group

Presented to the Society, November 11, 1966; received by the editors February 16, 1967.

( $\left.{ }^{1}\right)$ This research was supported by NSF GP-4007. 
operation and taking inverses, all in the case in which $R$ is quasi-local. Theorem 2 states that the convergent higher derivations form a subsemigroup $H_{c}(R, R)$ of $H(R, R)$ and Theorem 3 states that the subsets $H_{c}^{M}(R, R), H_{u}^{M}(R, R)$, and $H_{t}^{M}$, $(R, R)$ of $M$-convergent, uniformly $M$-convergent and strongly $M$-convergent higher derivations (see Definition 3) form subgroups of $H(R, R)$. An example following the proof of Theorem 2 illustrates the fact that $D$ may be convergent and $\alpha_{D}$ may be an automorphism whereas $D^{-1}$ is not convergent.

It is readily seen that if $D$ is $M$-convergent then $\alpha_{D}$ is in $H_{1}$, the subgroup of those inertial automorphisms $\alpha$ satisfying the condition $\alpha(a)-a \in M^{2}$ for all $a$ in $M$. Conversely, if $\alpha=\alpha_{D}$ and $\alpha$ is in $H_{1}$ then $D$ is $M$-convergent. If $R$ is a $v$-ring (unramified) every inertial automorphism is in $H_{1}$. If $R$ is an unramified complete regular local ring then the mapping $\Delta: D \rightarrow \alpha_{D}$ is a homomorphism of $H_{c}^{M}(R, R)$ onto $H_{1}$. As a matter of fact $\Delta$ restricted to $H_{t}^{M}(R, R)$ still maps onto $H_{1}$. It follows from work of Wishart [7, pp. 50,51] that a ramified $v$-ring may have inertial automorphisms represented by $D$ in $H_{c}^{M}(R, R)$ but not by $D$ in $H_{t}^{M}(R, R)$.

$\S$ III deals with the question of necessary and sufficient conditions on a complete local ring $R$ that every convergent higher derivation be uniformly convergent. Theorem 5 asserts that if the residue field $k$ has characteristic $p$, the condition that $k$ have a finite $p$-basis is sufficient and if $R$ is regular this condition is necessary. If $R$ is regular and $k$ has characteristic zero ( $R$ is a power series ring over $k$ ) then every convergent higher derivation is uniformly convergent if and only if $k$ has finite transcendency degree over its prime field.

II. Closure properties. Initially we assume $S$ to be an arbitrary associative ring and $R$ an over ring of $S$.

Definition 1. A higher derivation $D$ of $S$ into $R$ is a set $\left\{D_{i}\right\}_{i=1}^{\infty}$ of mappings of $S$ into $R$ such that for all $i \geqq 1$ and all $a, b$ in $S$,

(i) $D_{i}(a+b)=D_{i}(a)+D_{i}(b)$,

(ii) $D_{i}(a b)=\sum_{j=0}^{i} D_{j}(a) D_{i-j}(b)$,

where $D_{0}$ denotes the identity mapping. The symbol $H(S, R)$ will designate the set of all higher derivations of $S$ into $R$, and $Q$ will represent the higher derivation $\left\{Q_{i}\right\}$ such that $Q_{i}$ is the zero mapping for all $i \geqq 1$.

Definition 2. If $H$ and $D$ are in $H(S, S)$ then $K=H \circ D$ is the set of mappings $\left\{K_{i}\right\}_{i=1}^{\infty}$ where

$$
K_{i}=\sum_{j=0}^{1} H_{j} D_{i-j} .
$$

Proposition 1. The set of mappings $K$ as defined by (2) is a higher derivation.

Proof. Proposition 1 and Theorem 1, below, follow immediately from the following fact first observed by Schmidt [6]. Let $G$ represent the group of all automorphisms $\alpha$ on the power series ring $R[[X]]$ satisfying the conditions (i) $\alpha(X)=X$ and (ii) $\eta \alpha(a)=a$ for $a$ in $R$ where $\eta\left(\sum a_{i} X^{i}\right)=a_{0}$. Given $\alpha \in G$, 
$D^{\alpha}=\left\{D_{i}^{\alpha}\right\}$ is in $H(R, R)$ where $D_{i}^{\alpha}(a)$ is the coefficient of $X^{i}$ in $\alpha(a)$. The mapping $\alpha \rightarrow D_{\alpha}$ is a one-to-one correspondence between $G$ and $H(R, R)$ which then induces a group structure on $H(R, R)$, the induced operation being (2). Thus, we have

THEOREM 1. Given any ring $R, H(R, R)$ is a group with respect to the composition (2).

For later use we exhibit below an explicit description of $D^{-1}$ in terms of $D$. Let $(r, n)$ be a partition of the integer $n$ into $r$ nonnegative summands. If $(r, n)=i_{1}$, $\ldots, i_{r}$ we let $[D]_{(r, n)}$ be the sum of the formally distinct products of the $r$ maps $D_{i_{1}}, \ldots, D_{i_{r}}$. Thus, if $(3,5)=\{1,2,2\}$ then $[D]_{(3,5)}$ is $D_{1} D_{2}^{2}+D_{2} D_{1} D_{2}+D_{2}^{2} D_{1}$. Given $D$ in $H(R, R)$ we define $\bar{D}$ by

$$
\bar{D}_{n}=\sum_{(r, n)}(-1)^{r}[D]_{(r, n)}, \quad n \geqq 1,
$$

and contend that $\bar{D}=D^{-1}$.

The expression $\sum_{i=0}^{n} D_{i} \bar{D}_{n-i}$ is a sum of terms of the form $D_{j_{1}} \cdots D_{j_{r+1}}$ each such terms occuring twice in $D_{j_{1}} \bar{D}_{n-j_{1}}$ with coefficient $(-1)^{r}$ and in $D_{0} \bar{D}_{n}$ with coefficient $(-1)^{r+1}$. Hence $D \circ \bar{D}=Q$. But this equality uniquely determines the set of maps $\bar{D}$ and thus $\bar{D}=D^{-1}$.

LeMma 1. Let $(R, M)$ be a quasi-local ring and let $S$ be a subring of $R$ with the property that every nonunit of $S$ is in $M$. If $D$ in $H(S, R)$ converges then $D^{(i)}(S) \subset M$ for $i>0$.

Proof. Let $u$ be a unit in $S$ such that $D_{i}(u)$ is a unit for some $i>0$ and let $n$ be the least such integer. Since

$$
0=D_{n}(1)=D_{n}\left(u u^{-1}\right)=u D_{n}\left(u^{-1}\right)+u^{-1} D_{n}(u)+\sum_{i=1}^{n-1} D_{i}(u) D_{n-i}\left(u^{-1}\right)
$$

it follows that $D_{n}\left(u^{-1}\right)$ is also a unit. Since $D$ converges there is a largest integer, say $s$, such that $D_{s}(u)$ is a unit, and a largest integer $t$ such that $D_{t}\left(u^{-1}\right)$ is a unit. Now $0=D_{s+t}(1)=D_{s+t}\left(u u^{-1}\right)$ and $D_{s+t}\left(u u^{-1}\right)=D_{s}(u) D_{t}\left(u^{-1}\right), \bmod M$, which yields a contradiction. Thus $D_{i}(u)$ is in $M$ for all units $u$. Next, let $a$ be in $S \cap M$. Then $D_{i}(1+a)=D_{i}(1)+D_{i}(a)$ is in $M$ and thus $D_{i}(a)$ is in $M$. This proves Lemma 1 .

THEOREM 2. If $R$ is a quasi-local ring the set $H_{c}(R, R)$ of convergent higher derivations on $R$ is a subsemigroup of $H(R, R)$.

Proof. Let $D$ and $H$ be in $H_{c}(R, R)$. Given $a$ in $R$ and a positive integer $n$, there is an integer $m$ such that if $i \geqq m$ then $H_{i}(a)$ is in $M^{n}$ and there exists an integer $t$ such that if $i \geqq t$ then $D_{i} H_{j}(a)$ is in $M^{n}$ for $j=0,1, \ldots, m-1$. It is readily seen from (ii) of Definition 1 and from Lemma 1 that $D_{i}\left(M^{t}\right) \subset M^{t}$ for all $i>0$, and $t>0$. Thus, if $s$ is the maximum of $2 m$ and $2 t$ and if $j>s$ then $\sum_{i=0}^{j} D_{j} H_{i-j}(a)$ is in $M^{t}$. Thus $D \circ H$ is in $H_{c}(R, R)$. 
A simple example illustrates the fact that a convergent higher derivation need not have a convergent inverse. Let $k$ be any field and let $k[[X]]$ be the power series ring in the indeterminate $X$ over $k$. We define $D \in H(k[[X]], k[[X]])$ by the conditions

(i) $D_{j}(a)=0$ for $a \in k$ and all $j>0$;

(ii) $D_{1}(X)=X, D_{i}(X)=0$ for $i \geqq 2$.

These conditions determine an obviously unique higher derivation by [2, Theor $\epsilon \mathrm{m}$ 2] and Proposition 2 which appears later in this paper. We note that:

$$
D_{n}^{-1}(X)=\sum_{(r, n)}(-1)^{r}[D]_{(r, n)}(X)=(-1)^{n} D_{1}^{n}(X)=(-1)^{n} X .
$$

Since this is true for any $n>0$ it follows that $D^{-1}$ does not converge. Note, however, that $\alpha_{D}$ is an automorphism. As this example suggests a sufficient condition for $D \in H_{c}(R, R)$ to have a convergent inverse is that $D(M) \subset M^{2}$, by which is meant $D_{i}(M) \subset M^{2}$ for all $i>0$. We shall see (Lemma 5) that this condition is fullfilled if $R$ is a $v$-ring, a one-dimensional complete regular local ring having characteristic zero with residue field having characteristic $p \neq 0$.

Definition 3. Let $(R, M)$ be a quasi-local ring and let $S$ be a subring. $D$ in $H_{c}(S, R)$ is said to be

(a) $M$-convergent if $D(S \cap M) \subset M^{2}$;

(b) uniformly $M$ convergent if $D$ is $M$-convergent and converges uniformly;

(c) strongly convergent if $D_{i}(S) \subset M^{i}$ for $i=1,2, \ldots$ Strong $M$-convergence is defined as in (b).

The symbols $H_{u}(S, R)$ and $H_{t}(S, R)$ will represent the subsets of $H(S, R)$ consisting of the uniformly convergent $D$ and the strongly convergent $D$ respectively. A superscript $M$ indicates $M$ convergence i.e. $H_{u}^{M}(S, R)$ is the set of all uniformly $M$ convergent $D$ in $H(S, R)$.

THEOREM 3. Let $R$ be a quasi-local ring. $H_{c}^{M}(R, R), H_{u}^{M}(R, R)$ and $H_{t}^{M}(R, R)$ are all subgroups of $H_{c}(R, R) . H_{u}(R, R)$ is a subsemigroup of $H_{c}(R, R)$.

Proof. Obviously the product of $M$-convergent higher derivations is $M$ convergent. We note that if $D$ and $H$ of the proof of Theorem 2 are in $H_{u}(R, R)$ then the proof is independent of the choice of $a$ and hence $D \circ H$ is in $H_{u}(R, R)$. If $D$ is in $H_{t}^{M}(R, R)$ then

$$
D_{i}\left(M^{j}\right) \subset M^{i+j}, \quad i \geqq 1, \quad u \geqq 0 .
$$

Relation (4) implies closure in $H_{t}^{M}(R, R)$ and also leads immediately to the conclusion that if $(r, i)$ is any partition of $i$ and $D \in H_{t}^{M}(R, R)$ then $[D]_{(r, i)}\left(M^{j}\right) \subset M^{i+j}$. Thus if $D$ is in $H_{t}^{M}(R, R)$ so is $D^{-1}$. The example following Theorem 2 is a strongly convergent higher derivation. If $D$ represents the higher derivation in question and $H=D \circ D$ then $H_{2}(X)=X$, illustrating the fact that $H_{t}(R, R)$ is neither closed with respect to product nor with respect to taking inverse. 
In order to verify that the inverse of $D$ in $H_{c}^{M}(R, R)$ is in $H_{c}^{M}(R, R)$ it is sufficient to show that, given $a$ in $R$ and $m \geqq 0$, there is an integer $n$ such that if $i_{1}, \ldots, i_{r}$ is any partition into positive integers of $t>n$ then,

$$
D_{i_{1}} \cdots D_{i_{r}}(a) \in M^{m} \text {. }
$$

Since for $D$ in $H_{c}^{M}(R, R)$

$$
D_{i}\left(M^{j}\right) \subset M^{j+1}, \quad i>0, \quad j \geqq 0
$$

it follows that (5) holds if $r \geqq m$. There is an integer $n_{1}$ such that if $i>n_{1}$ then $D_{i}(a) \in M^{m}$ and an integer $n_{2}$ such that if $i_{2}>n_{2}$ then $D_{i_{2}} D_{i_{1}}(a) \in M^{m}$ for $i_{1}=1,2$, $\ldots, n_{1}$.

Iteratively, we define integers $n_{1}, n_{2}, \ldots, n_{m-1}$ such that, if $0<j<m$ and $i_{j}>n_{j}$, then $D_{i_{1}} D_{i_{j-1}}, \ldots, D_{i_{1}}(a) \in M^{m}$ if $0<i_{t} \leqq n_{t}$ for $t=1, \ldots, j-1$. Let $n^{\prime}$ be the maximum of $n_{1}, n_{2}, \ldots, n_{m-1}$ and let $n=m\left(n^{\prime}+1\right)$. If $j_{1}, \ldots, j_{r}$ are positive integers such that $j_{1}+\cdots+j_{r}>n$ then either $r \geqq m$ or $j_{t}>n^{\prime}$ for some $t$. In either case $D_{i_{1}}, \ldots, D_{i_{r}}(a) \in M^{m}$. It follows then from (3) that if $D$ is $M$ convergent so is $D^{-1}$. If $D$ is in $H_{u}^{M}(R, R)$ the above argument again applies independently of the choice of $a$. We conclude that $D^{-1}$ is in $H_{u}^{M}(R, R)$ if $D$ is in $H_{u}^{M}(R, R)$.

III. Uniformly convergent higher derivations. We begin with some basic facts about extensions of higher derivations and their convergence properties. Let $T$ be a commutative overring of a ring $S$ and let $a \in S$ be invertible in $S$. Then if $D \in H(S, T)$

$$
D_{n}\left(a^{-1}\right)=\sum_{(r, n)}(-1)^{r+1} a^{-(r+1)} C(r, n)[D(a)]_{i r, n)}
$$

where $C(r, n)=r ! /\left(n_{1} ! \cdots n_{t} !\right)$ and $n_{1}, \ldots, n_{t}$ represent the number of times the distinct integers of $(r, n)$ occur in $(r, n)$. Also if $(r, n)=j_{1}, \ldots, j_{r}$ then $[D(a)]_{(r, n)}$ is the sum of all the formally distinct products of the $r$ quantities $D_{j_{1}}(a), \ldots, D_{j_{r}}(a)$. For $n=1$ we have $D_{1}\left(a^{-1}\right)=-a^{-2} D_{1}(a)$. Proceeding by induction, $0=D_{n}\left(a a^{-1}\right)$ $=\sum D_{i}(a) D_{n-i}\left(a^{-1}\right)$ or $D_{n}\left(a^{-1}\right)=-a^{-1} \sum_{i=0}^{n-1} D_{n-i}(a) D_{i}\left(a^{-1}\right)$. Substitution of $(6, i)$ in the right hand side of this equality for $i=1, \ldots, n-1$ yields $(6, \mathrm{n})$ without difficulty. Let $T$ and $S$ be as above and let $D$ be in $H(S, T)$. The mapping $\tau_{D}: S \rightarrow T[[X]]$ given by (7) is an isomorphism with the property $\eta \tau_{D}$ is the

$$
\tau_{D}(a)=\sum_{i=0}^{\infty} D_{i}(a) x^{i}
$$

identity on $S$ where again $\eta\left(\sum a_{i} X^{i}\right)=a_{0}$. Conversely, if $\tau: S \rightarrow T[[X]]$ is a homomorphism such that $\eta \tau$ is the identity on $S$ then $\tau(a)=a+\sum X^{i} D_{i}^{\tau}(a)$ and $D^{\tau}=\left\{D_{i}^{\tau}\right\}$ is in $H(S, T)$. As in the proof of Theorem $1, D \rightarrow \tau_{D}$ is a one-to-one correspondence between $H(S, T)$ and the set of isomorphisms $\tau$ of $S$ into $T[[X]]$ such that $\eta \tau$ is the identity map on $S$.

Let $M$ be a multiplicatively closed subset of $S$ each element of which has an inverse in $T$. Thus $S_{M}$ the ring of quotients with respect to $M$ is a subring of $T$.

Lemma 2. Each $D$ in $H(S, T)$ has a unique extension to $H\left(S_{M}, T\right)$. 
Proof. The lemma follows from the existence and uniqueness of the extension of $\tau_{D}$ to $S_{M}$.

LEMMA 3. Let $S$ be a subring of the quasi-local ring $(R, M)$ and let $B$ be a subset of $R$. Let $D$ be in $H(S[B], R)$.

(i) If $D$ converges on $S$ and on $B$ then $D \in H_{c}(S[B], R)$.

(ii) If $D$ is uniformly convergent on $S$ and on $B$ and $D(S[B]) \subset M$ then $D \in H_{u}(S[B], R)$.

(iii) If $D$ is strongly convergent on $S$ and on $B$ then $D \in H_{t}(S[B], R)$.

(iv) If $D(S \cap M) \subset M^{2}$ and $D(B \cap M) \subset M^{2}$ then $D(S[B] \cap M) \subset M^{2}$.

Proof. Each element in $S[B]$ is a sum of terms of the form $s b_{1} \cdots b_{t}$ where $s \in S ; b_{1}, \ldots, b_{t} \in B$ and $t \geqq 0$. Now

$$
D_{n}\left(s, b_{1}, \ldots, b_{t}\right)=\sum_{i_{0}+\cdots+i_{t}=n} D_{i_{0}}(s) D_{i_{1}}\left(b_{1}\right) \cdots D_{i_{t}}\left(b_{t}\right) .
$$

Clearly, if $D$ converges at $s, b_{1}, \ldots, b_{t}$ then $D$ converges at $s b_{1} \cdots b_{t}$.

Statement (ii) is a consequence of the following lemma which will be useful elsewhere.

LEMMA 4. Let $S$ be a subring of a quasi-local ring $(R, M)$ and let $B$ be a subset of $S$. If $D \in H(S, R)$ converges uniformly on $B$ and $D(B) \subset M$ then given $n>0$, there is an $m>0$ such that given any product $b_{1} \cdots b_{t}$ of $t \geqq 1$ elements in $B, D_{i_{1}}\left(b_{1}\right) \cdots$ $D_{i_{t}}\left(b_{t}\right) \in M^{n}$ whenever $i_{1}+\cdots+i_{t}>m$.

Proof. There is an integer $r$ such that if $i>r$, then $D_{i}(B) \subset M^{n}$. Let $m=n r$. Then, if $i_{1}+\cdots+i_{t}>m$ either $n$ of the $i$ 's are different from zero or one of them is greater than $r$. In either case $D_{i_{1}}\left(b_{1}\right) \cdots D_{i_{t}}\left(b_{t}\right)$ is in $M^{n}$.

To prove (iii) of Lemma 3 we simply observe that if $D_{i}(a) \in M^{i}$ for $a$ in $S$ or in $B$ then (8) is in $M^{n}$. Statement (iv) is immediate.

Corollary 3.1. If $D \in H_{c}(S[B], R)$ converges uniformly on $S$, where $B$ is a finite set and $D(S[B]) \subset M$, then $D \in H_{u}(S[B], R)$.

Corollary 3.2. Let $M$ be a multiplicatively closed subset of $S$ each element of which has an inverse in $R$ and let $\bar{D} \in H\left(S_{M}, R\right)$ be the extension of $D \in H(S, R)$. If $D(S) \subset M$, it follows that

(i) if $D \in H_{c}(S, R)$ then $\bar{D} \in H_{c}\left(S_{M}, R\right)$;

(ii) if $D \in H_{u}(S, R)$ then $\bar{D} \in H_{u}\left(S_{M}, R\right)$;

(iii) if $D \in H_{t}(S, R)$ then $\bar{D} \in H_{t}\left(S_{M}, R\right)$.

Proof. Let $M^{-1}$ denote the set of inverses of the elements of $M$. Then $\bar{D}\left(M^{-1}\right)$ $\subset M$ in view of (6) and the assumption that $D(S) \subset M$. Also, it follows from Lemma 4 and (6) that if $D \in H_{c}(S, R)$ then $\bar{D}$ converges on $M^{-1}$, and if $\bar{D} \in H_{u}(S, R)$ then $\bar{D}$ converges uniformly on $M^{-1}$. If $D \in H_{t}(S, R)$ it is apparent from (6) that $D$ is strongly convergent on $M^{-1}$. The observation that $S_{M}=S\left[M^{-1}\right]$ and an appeal to Lemma 3 completes the proof. 
The symbol $V$ will represent a valuation ring having characteristic zero with residue field $k$ of characteristic $p \neq 0$. Let $\pi$ be a prime element of $V$ and let $e$ be the ramification of $V$, that is $p V=\pi^{e} V$, and we write $e=p^{s} r$ where $(p, r)=1$. Let $(R, M)$ be a regular local ring containing $V$ such that $\pi V=V \cap M$.

Lemma 5. Each $D$ in $H_{c}(V, R)$ has the property $D(\pi V) \subset M^{2}$ and thus $H_{c}(V, R)$ $=H_{c}^{M}(V, R)$.

Proof. For some positive integer $t, \pi V \subset M^{t} \backslash M^{t+1}$, i.e. $\pi V \subset M^{t}$ but $\pi V \notin M^{t+1}$. Thus $\pi \in M^{t} \backslash M^{t+1}$. Let $i$ be the least integer such that $D_{i}(\pi) \notin M^{2}$. We assume $t>1$. Now

$$
D_{i r}\left(\pi^{r}\right)=\left[D_{i}(\pi)\right]^{r}+\sum_{i_{1}+\cdots+i_{r}=i r ; \text { some } i_{j}<i} D_{i_{1}}(\pi), \ldots, D_{i_{r}}(\pi) .
$$

Since $\left[D_{i}(\pi)\right]^{r} \in M^{r} \backslash M^{r+1}$ and the second term is seen to be in $M^{r+1}$ we have $D_{i r}\left(\pi^{r}\right) \in M^{r} \backslash M^{r+1}$. Similarly,

$$
\text { (9) } \quad D_{p^{s} i r}\left(\pi^{p^{s} r}\right)=\left[D_{i r}\left(\pi^{r}\right)\right]^{p^{s}}+\sum_{i_{1}+\cdots+i_{p s}=p^{s} i r ; i_{j} \neq i_{k} \text { for some } j, k \text {. }} D_{i_{1}}\left(\pi^{r}\right) \cdots D_{i_{p} s}\left(\pi^{r}\right) \text {. }
$$

Again $\left[D_{i r}\left(\pi^{r}\right)\right]^{p^{s}} \in M^{r p^{s}} \backslash M^{r p^{s+1}}$ and the remaining term on the right of (9) is seen to be in $M^{r p^{s+1}}$ since each summand occurs a multiple of $p$ times. We conclude from (9) that

$$
D_{p^{s} i r}\left(\pi^{p^{s} r}\right) \in M^{p^{s} r} \backslash M^{p^{s} r+1} .
$$

For some unit $u$ in $V p=u \pi^{p^{s} r}$ and

$$
0=D_{p^{s} i r}(p)=u D_{p^{s} i r}\left(\pi^{p^{s} r}\right)+\sum_{j=1}^{p_{i i r-j}^{j}} D_{j}(u) D_{p^{s_{i r-j}}}\left(\pi^{p^{s} r}\right) .
$$

By an argument like that above applied to the right side of (10) we conclude that $D_{p^{s}{ }_{i r}}(p) \in M^{p^{s} r} \backslash M^{p^{s}+1}$ which is the desired contradiction.

If $t=1$ then we observe as above that $D_{i}\left(\pi^{r}\right) \in M^{r} \backslash M^{r+1}$ and hence that $D_{p^{s_{i}}}\left(\pi^{p^{s} r}\right) \in M^{p^{s} r} \backslash M^{p^{s} r+1}$. It follows that $D_{p_{i}}(p)=D_{p_{i}}\left(u \pi^{p^{s} r}\right) \notin M^{p^{s} r+1}$; a contradiction. This proves Lemma 5.

Lemma 6. If $D$ is in $H(V, R)$ and $a$ is in $V$ then $D_{i}\left(a^{p^{n}}\right) \subset M^{j}$ for $i<p^{n-j}$.

Proof. We note that

$$
\begin{aligned}
D_{i}\left(a^{p^{n}}\right) & =\sum_{i_{1}+\cdots+i_{p n}=i} D_{i_{1}}(a) \cdots D_{i_{p} n}(a) \\
& =C\left[p^{n} ; q_{1}, \ldots, q_{t}\right]\left[D_{j_{1}}(a)\right]^{q_{1}}, \ldots,\left[D_{j_{t}}(a)\right]^{q_{t}}
\end{aligned}
$$

where the set $i_{1}, \ldots, i_{p_{n}}$ consists of $q_{r}$ integers $j_{r}$ for $r=1, \ldots, t$ and $C\left[p^{n} ; q_{1}, \ldots, q_{t}\right]$ is the indicated multinomial coefficient. Since $i<p^{n-j}$, and hence $q_{r}<p^{n-j}$ for at least one $q_{r}$, it follows that the maximum integer $t$ such that $p^{t} \mid q_{r}$, for all $q_{r}$ is less than $n-j$. Thus $p^{j} \mid C\left[p^{n} ; q_{1}, \ldots, q_{t}\right]$. (Here we are using the fact that if $s$ is the largest integer such that $p^{s} \mid q_{r}$ for all $r$ then $p^{n-s} \mid C\left[p^{n} ; q_{1}, \ldots, q_{t}\right]$.) It follows from (11) that $D_{i}\left(a^{p^{n}}\right) \subset M^{j}$. 
We now make an additional assumption on $V$ and $R$, namely that $R$ is complete in the $M$-adic topology and $V$ is a complete subring with $e=1$.

THEOREM 4. Let $\bar{S}$ be a p-basis for $k$ the residue field of $V$ and let $S \subset V$ be a set of representatives of the elements in $S$. If $f$ is a mapping of $S \times I$ into $R$ where $I$ denotes the positive integers then

(a) There is one and only one $D \in H(V, R)$ with the property $D_{\imath}(s)=f(s, i)$ for $(s, i)$ in $S \times I$.

(b) $D$ is in (i) $H_{c}^{M}(V, R)$, (ii) $H_{u}^{M}(V, R)$, (iii) $H_{t}^{M}(V, R)$ if and only if $D(S) \subset M$ and (i) $D$ converges on $S$, (ii) $D$ converges uniformly on $S$, (iii) $D_{i}(S) \subset M^{i}$ for $i=1$, $2, \ldots$

Proof. In order to prove part (a) we consider $V_{0}$ the complete subring of $V$ having residue field $k_{0}$, the maximal perfect subfield of $k$. Since $\bar{S}$ is an algebraically independent set over $k_{0}, S$ is algebraically independent over $V_{0}$. Thus by a standard Zorn's Lemma argument using [2, Theorem 2] we can define $H \in H\left(V_{0}(S), R\right)$ by the conditions (i) $H$ restricted to $V_{0}$ is the zero higher derivation and (ii) $H_{i}(s)=f(s, i)$ for $s \in S$ and $i \in I$.

Let $\bar{U}$ be a basis for $k$ as a linear space over $k_{0}(\bar{S})$ and let $U$ be a set of representatives in $V$ of the elements in $\bar{U}$. We assume that 1 is in $U$.

The set $\bar{U}^{p^{n}}$ of $p^{n}$ th powers of elements of $\bar{U}$ is also a basis for $k$ over $k_{0}(\bar{S})$ [3, p. 347]. If $V_{0}(S)$ is the ring of rational functions over $V_{0}$ in the elements of $S$ then $V_{1}=V_{0}(S) \cap V$ is a valuation ring with residue field $k_{0}(S)$. Thus, given $a \in V$, there are elements $a_{1}, \ldots, a_{n}$ in $V_{1}$ and $u_{1}, \ldots, u_{n}$ in $U$ such that

$$
a=\sum a_{i} u_{i}^{p^{n}}, \quad \bmod p^{n} .
$$

Moreover, the $a_{i}$ are uniquely determined, $\bmod p^{n}$, by the condition (12).

For $i=1, \ldots, m$ and $a \in V$, let

$$
D_{i}^{(m)}\left(a+p^{m} V\right)=\sum H_{i}\left(a_{j}\right) u_{j}^{p^{3 m}}+M^{m},
$$

where $a=\sum a_{j} u_{j}^{p^{3 m}}, \bmod p^{m}$, according to (12). The fact that the $a_{j}$ are determined, $\bmod p^{n}$, assures that $D_{i}^{(m)}$ is a well defined map of $V / p^{m} V$ into $R / M^{m}$. We define the desired $D \in H(V, R)$ by the coset intersection

$$
D_{i}(a)=\bigcap_{m>i} D_{i}^{(m)}\left(a+p^{m} V\right)
$$

The following equalities which will be verified in turn, permit us to conclude that $D$ is a higher derivation. For $A$ and $B$ in $V / p^{m} V$

$$
\begin{aligned}
D_{i}^{(m)}(A+B) & =D_{i}^{(m)}(A)+D_{i}^{(m)}(B) \quad \text { for } i=1, \ldots, m, \\
D_{i}^{(m)}(A B) & =\sum_{j=0}^{i} D_{j}^{(m)}(A) D_{i-j}^{(m)}(B)
\end{aligned}
$$

and for $a \in V$ the following coset inclusion holds.

$$
D_{i}^{(m)}\left(a+p^{m} V\right) \supset D_{i}^{(m+1)}\left(a+p^{m+1} V\right) .
$$


Statement (15) is clear from the definition. In order to establish (16) we let $A=\sum a_{k} u_{k}^{p^{3 m}}+p^{m} V$ and $B=\sum b_{j} u_{j}^{p^{3 m}}+p^{m} V$, using (12). Thus

$$
A B=\sum a_{k} b_{j} u_{k}^{p^{3 m}} u_{j}^{p^{3 m}}+p^{m} V .
$$

Using (12) we have $u_{k} u_{j}=\sum d_{r} u_{r}, \bmod p V$. Thus [2, Lemma 1],

$$
u_{k}^{p^{3 m}} u_{j}^{p^{3 m}}=\sum_{t=0}^{3 m-1} p^{t} \sum_{l} s_{k, j, t, l} c_{k, j, t, l}^{p^{3 m-t}} u_{l}^{p^{3 m}}, \quad \bmod p^{3 m} V
$$

where $s_{k, j, t, l}$ is a rational integer and $c \in V_{1}$. Substituting (19) into (18) we have

$$
D_{i}^{(m)}(A B)=\sum H_{i}\left(\sum a_{k} b_{j} p^{t} s_{k, j, t, l} c_{k, j, t, l}^{p^{3 m-t}}\right) u^{p^{3 m}}+M^{m} .
$$

Since $p$ and $s_{k, j, t, l}$ are rational integers $H_{i}\left(p^{t}\right)=H_{i}\left(s_{k, j, t, l}\right)=0$, for all $i$. Also, by Lemma $6, H_{i}\left(c_{k, j, t, l}^{p^{3 m-t}}\right)$ is in $M^{m}$ if $t<m$, since $i \leqq m$. Thus, $\bmod M^{m}$, we have

$$
\begin{aligned}
H_{i}\left(\sum a_{k} b_{j} p^{t} s_{k, j, t, l} c_{k, j, t, l}^{p^{3 m-t}}\right) & =\sum H_{i}\left(a_{k} b_{j}\right) p^{t} s_{k, j, t, l} c_{k, j, t, l}^{p^{3 m-t}} \\
& =\sum \sum_{r=0}^{i} H_{r}\left(a_{k}\right) H_{i-r}\left(b_{j}\right) p^{t} s_{k, j, t, l} c_{k, j, t, l}^{p^{3 m-t}} .
\end{aligned}
$$

Thus, substituting this last expression into (20) and then using (19) we find that (20) reduces to $\sum H_{r}\left(a_{k}\right) H_{i-r}\left(b_{j}\right) u_{k}^{p^{3 m}} u_{j}^{p^{3 m}}+M^{m}$ from which (16) follows.

Relation (17) can be verified as follows. Using (12) for $n=1$ we have $u_{k}^{p^{3}}=\sum a_{i} u_{i}$, $\bmod p$, the $a_{i}$ being in $V_{1}$. Thus [2, Lemma 1]

$$
u_{k}^{p^{3(m+1)}}=\left[\sum a_{i} u_{i}\right]^{p^{3 m}}=\sum_{t=0}^{3 m-1} p^{t} \sum s_{k, t, n} c_{k, t, n}^{p^{3 m-1}} u_{n}^{p^{3 m}}, \quad \bmod p^{3 m} V .
$$

Again, $s_{k, t, n}$ is a rational integer and $c_{k, t, n} \in V_{1}$. Thus if $a+p^{m+1} V=\sum a_{k} u_{k}^{p^{3(m+1)}}$ $+p^{m+1} V$ then

$$
\begin{aligned}
D_{i}^{(m+1)}\left(a+p^{m+1} V\right) & =\sum H_{i}\left(a_{k}\right) u_{k}^{p^{3(m+1)}}+M^{m+1} \\
& =\sum H_{i}\left(a_{k}\right) \sum_{t=0}^{3 m-1} p^{t} \sum s_{k, t, n} c_{k, t, n}^{p^{3 m-t}} u_{n}^{p^{3 m}}+M^{m+1} \\
& =\sum H_{i}\left(a_{k} \sum p^{t} \sum s_{k, t, n} c_{k, t, n}^{p^{3 m-t}}\right) u_{n}^{p^{3 m}}+M^{m+1}
\end{aligned}
$$

But, $a+p^{m} V=\sum\left(a_{k} \sum p^{t} \sum s_{k, t, n} c_{k, t, n}^{p^{3 m-t}}\right) u_{n}^{p^{3 m}}+p^{m} V$ and

$$
D_{i}^{(m)}\left(a+p^{m} V\right)=\sum H_{i}\left(a_{k} \sum p^{t} s_{k, t, n} c_{k, t, n}^{p^{3 m-t}}\right) u_{n}^{p^{3 m}}+M^{m} .
$$

Relation (17) then follows in view of (22).

Since $\bigcap_{n=1}^{\infty} M^{n}=0, D_{i}$ as defined by (14) is a uniquely determined element of $R$. Properties (15) and (16) assure that conditions (i) and (ii) of Definition 1 hold $\bmod M^{m}$ for all $m$. Thus $D$ is a higher derivation.

In order to show that $D$ is an extension of $H$ we note that if $a \in V_{1}$ then $D_{i}^{(m)}(a)=H_{i}(a)+M^{m}$ since $1 \in U$. Thus $D_{i}(a)=\bigcap_{m} D_{i}^{(m)}\left(a+p^{m} V\right)=H_{i}(a)$. 
It remains to show that $D$ is determined by $W=\left\{D_{i}(s)\right\}_{i=1, s \in S}^{\infty}$. Certainly, the restriction of $D$ to $V_{1} \subset V_{0}(S)$ is completely determined by $W$ since $D_{i}(a)=0$ for $i>0$ and $a$ in $V_{0}$ by Lemma 6 and the fact that $V_{0}$ is for each $n>0$ the completion of the subring generated by the $p^{n}$ th powers of elements in $V_{0}$. Let $a$ be any element in $V$. By (12) $a=\sum a_{i} u_{i}^{p^{3 m}}, \bmod p^{3 m+1}$, where the $a_{i}$ are in $V_{1}$. If $j<m$,

$$
D_{j}\left(\sum a_{i} u_{i}^{p^{3 m}}\right)=\sum D_{j}\left(a_{i}\right) u_{i}^{p^{3 m}}, \quad \bmod M^{m},
$$

by Lemma 6 . Hence $D,(a)=\sum D_{j}\left(a_{i}\right) u_{i}^{p^{3 m}}, \bmod M^{m}$. Thus $D$ is determined, $\bmod M^{m}$ by its restriction to $S$. But $m$ is arbitrary. It follows that $D$ is uniquely determined by its action on $S$. This proves (a) of Theorem 4 .

If $D$ in $H(V, R)$ converges then $D(V) \subset M$. Hence the condition $D(S) \subset M$ is necessary for $D$ to be in $H_{c}^{M}(V, R), H_{u}^{M}(V, R)$ or $H_{t}^{M}(V, R)$. The remaining condition is clearly necessary in each case.

Let $D$ in $H(V, R)$ be such that $D(S) \subset M$ and $\sum D_{i}(s)$ converges for all $s \in S$. To show that $D$ is in $H_{c}^{M}(V, R)$ it is only necessary to show that $D$ converges in view of Lemma 5. Given $n>0$. By Lemma $6 D_{j}\left(V^{p^{n+1}}\right) \subset M$ for $j \leqq n$. But $V=V^{p^{n+1}}[S]+p V$ and hence $D,(V) \subset M$ or

$$
D(V) \subset M \text {. }
$$

LEMMA 7. Let $(T, M)$ be a quasi-local ring with residue field having characteristic $p \neq 0$. Let $S$ be a subring of $T$. If $D \in H(S, T)$ maps $S$ into $M$ then

$$
D\left(S^{p^{n}}\right) \subset M^{n+1}, \quad \text { for } n=1,2, \ldots
$$

Proof. We argue by induction on $n$ using

$$
D_{i}\left(a^{p}\right)=p a^{p-1} D_{i}(a)+\sum_{i_{1}+\cdots+i_{p}=i ; i_{j}<i} D_{i_{1}}(a) \cdots D_{i_{p}}(a) .
$$

Since at least two of the integers $i_{1}, \ldots, i_{p}$ are different from zero $D_{i}\left(a^{p}\right)$ is in $M^{2}$. If in (25) $a=b^{p^{n}}$ then, by induction, $D\left(b^{p^{n}}\right) \in M^{n+1}$ and hence $D_{1}\left(b^{p^{n+1}}\right) \in M^{n+2}$.

By relation (23) and Lemma 7 then $D\left(V^{p^{n}}\right) \subset M^{n+1}$. Given $a$ in $V$ and $t>0$, $a=f\left(s_{1}, \ldots, s_{q}\right), \bmod p^{t} V$, where $f \in V^{p^{t}}\left[X_{1}, \ldots, X_{q}\right]$ has degree $<p^{t}$ in each $X_{i}$, and $\left\{s_{1}, \ldots, s_{q}\right\} \subset S$. We choose $n$ so that if $i>n / q p^{t}$ then $D_{i}\left(s_{j}\right) \in M^{t}$ for $j=1, \ldots, q$.

$$
D_{i}\left(b s_{1}^{n_{1}} \cdots s_{q}^{n_{q}}\right)=\sum_{i_{0}, \ldots, i_{n_{1}}+\ldots+n_{q}=i} D_{i_{0}}(b) D_{i_{1}}\left(s_{1}\right) \cdots D_{i_{n_{1}}+\cdots+n_{q}}\left(s_{q}\right) .
$$

If $i>n$ in (26) either $i_{0}>0$ or $i_{j}>n / q p^{t}$ for some $j>0$. Thus, since $b \in V^{p^{t}}$, $D_{i_{0}}(b) D_{i_{1}}\left(s_{1}\right) \cdots D_{i_{n_{1}}+\ldots+n_{q}}\left(s_{q}\right)$ is in $M^{t}$. Since every term in $V^{p^{t}}\left[s_{1}, \ldots, s_{q}\right]$ is of the type treated in (26) it follows that, if $i>n, D_{i}(a) \in M^{t}$. Thus $D$ converges.

If $D$ converges uniformly on $S$ then the $n$ of the previous paragraph can be chosen so that if $i>n / q p^{t}$ then $D_{i}(S) \subset M^{t}$, from which it follows that $D_{i}(V)$ $=D_{i}\left(V^{p^{t}}[S]+p^{t} V\right) \subset M^{t}$.

Thus $D \in H_{u}^{M}(V, R)$. Similarly, if $D_{i}(S) \subset M^{i}$ a like argument leads to the conclusion that $D_{i}(V) \subset M^{i}$. 
THEOREM 5. If $(R, M)$ is a complete local ring with residue field $k$ having characteristic $p \neq 0$ then $H_{c}(R, R)=H_{u}(R, R)$ if $k$ has a finite $p$-basis. If $R$ is regular $H_{c}(R, R)=H_{u}(R, R)$ only if $k$ has a finite p-basis.

Proof. As in Theorem 4 we let $S$ be a set of units in $R$ which map biuniquely onto a $p$-basis $\bar{S}$ for $k$ under the canonical map of $R$ onto $k$. It is assumed that $\bar{S}$ is finite. Let $\mathscr{M}$ be the set of multiplicative representatives of the element in $k_{0}$, the maximal perfect subfield of $k$. We choose an arbitrary $D$ in $H_{c}(R, R)$ and observe first that $D(\mathscr{M})=\{0\}$, by Lemma 7 since each $a$ in $\mathscr{M}$ is a $p^{m}$ th power for all $m$. Thus if $T$ is the subring of $R$ generated by $\mathscr{M}$ then $\left.D\right|_{T}$, the restriction of $D$ to $T$ is the zero higher derivation. By Corollary 3.1 $\left.D\right|_{T[S]}$ is uniformly convergent.

Let $U$ be a subset of $R$ which maps biuniquely onto $\bar{U}$ a basis for $k$ as a linear space over $k_{0}(S)$. As we have observed before the set $U_{n}$ of $p^{n}$ th powers of the elements in $U$ maps onto a basis for $k$ over $k_{0}(\bar{S})$.

Let $t>0$ be fixed. If $M=\sum_{i=1}^{s} w_{i} R$, then $a \in R \Rightarrow$ :

$$
a=\sum_{i} f_{i} u_{i}^{p^{t}}+\mu, \quad \mu \in M^{t}, \quad f_{i} \in T[S]\left[w_{1}, \ldots, w_{s}\right] .
$$

Hence applying Corollary 3.1 to obtain $D \mid T[S]\left[w_{1}, \ldots, w_{s}\right]$ uniformly convergent, we pick an $n$ such that $j>n$ implies

$$
D_{j}\left(T[S]\left[w_{1}, \ldots, w_{s}\right]\right) \subset M^{t} .
$$

Thus since $D\left(M^{t}\right) \subset M^{t}$,

$$
D_{j}(a)=D_{j}\left(\sum_{i} f_{i} u_{i}^{p^{t}}\right)+D_{j}(\mu) \in M^{t} .
$$

Since the choice of $n$ depends only on $t, S$, and $\left\{w_{1}, \ldots, w_{s}\right\}$ it follows that $D$ converges uniformly on $R$. Inclusion the other way is obvious so the first part of Theorem 5 is proved.

In proving the rest of Theorem 5 we will have use for the following proposition whose proof is standard and will be omitted.

Proposition 2. Let $S$ be a subring of a complete local ring $(R, M)$ and let $D$ be in $H(S, R)$. If $D$ is continuous in the induced topology then $D$ extends and in only one way to a higher derivation $D^{*}$ on $S^{*}$ the completion of $S$ in $R$. If $D$ is convergent so is $D^{*}$. If $D$ is uniformly convergent so is $D^{*}$. If $D(S) \subset M$ then $D^{*}\left(S^{*}\right) \subset M$.

Assuming $R$ to be regular we consider the converse. If $R$ has characteristic $p$ then $R$ is a power series ring $k\left[\left[X_{1}, \ldots, X_{n}\right]\right]$ in a finite number of indeterminates $X_{1}, \ldots, X_{n}$ over its residue field $k$. We assume that $k$ possesses a $p$-basis $S$ with infinite cardinal. Let $\left\{s_{i}\right\}_{i=1}^{\infty}$ be a countable sequence of elements in $S$. A higher derivation $D^{(i)}$ in $H\left(k, k\left[\left[X_{1}, \ldots, X_{n}\right]\right]\right)$ is uniquely determined by the conditions (i) $D_{j}^{(i)}\left(s_{i}\right)=\delta_{i, j}$ (ii) $D_{j}^{(i)}(s)=0$ for $j \geqq 1$ and $s \in S, s \neq s_{i}$ [2, Theorem 1]. The theorem referred to here applies to $D \in H(k, k)$ but the proof applies to the case in which 
the range of $D$ is a ring containing $k$. Let $H^{(i)}$ be defined by $H_{n i}^{(i)}=X_{1}^{n} D_{n}^{(i)}, n \geqq 1$, and $H_{m}^{(i)}=\theta$, for $m$ not a multiple of $i, \theta$ being the zero map. $H^{(i)}$ so defined is a convergent higher derivation. $H^{(i)}$ is extended to $H^{(i)}$ on $k\left[X_{1}, \ldots, X_{n}\right]$ by the condition $H_{j}^{(i)}\left(X_{t}\right)=0$ for $j \geqq 1$, and $t=1, \ldots, n . H^{(i)}$ extended is again, by Lemma 3 , a convergent higher derivation. Finally, let $E=H^{(1)} \circ H^{(2)} \circ \ldots \circ H^{(n)} \circ \ldots$. Thus $E_{n}=\left(H^{(1)} \circ \ldots \circ H^{(n)}\right)_{n}$ since $H_{m}^{(i)}=\theta$ for $m<i$. It follows readily that $E$ is a welldefined higher derivation, and is clearly convergent. Let $E^{*}$ represent the extension of $E$ to $k\left[\left[X_{1}, \ldots, X_{n}\right]\right]$. Again by Proposition $2, E^{*}$ is a convergent higher derivation. It follows immediately from the definition of $E^{*}$ that $E_{i}^{*}\left(s_{i}\right)$ is in $M$ and not in $M^{2}$. Hence, $E^{*}$ is not uniformly convergent.

Assume now that $R$ has characteristic zero. Then $R=R_{1}[\pi]$ where

$$
R_{1}=V\left[\left[X_{1}, \ldots, X_{n}\right]\right]
$$

is a power series ring in $n$ indeterminates over an unramified $v$-ring $V$ and $\pi$ is a root of an Eisenstein polynomial $f$ over $R$ [1, Theorem 1].

The following facts will be useful. Let $K$ be the quotient field of $R_{1}$.

(A) A given higher derivation on $R_{1}$ has a unique extension to a higher derivation on $K$. This follows from Lemma 2.

(B) A higher derivation $D$ on $K$ has a unique extension $\bar{D}$ to $K[\pi]$ [2, Theorem 3]. If $D$ is convergent on $K, \bar{D}$ will be convergent if and only if $\sum \bar{D}_{i}(\pi)$ converges. If $D\left(R_{1}\right) \subset R$ then $\bar{D}(R) \subset R$ if and only if $\bar{D}(\pi) \in R$.

Let the minimal polynomial $f$ of $\pi$ over $R$, be $f=X^{e}+f_{e-1} X^{e-1}+\cdots+f_{0}$ and let $f^{\prime}$ denote the ordinary derivative of $f$.

LEMMA 8. If $f^{\prime}(\pi) \in M^{t} \backslash M^{t+1}$ and $D \in H_{c}\left(R_{1}, R_{1}\right)$ is such that $D\left(f_{j}\right) \in M^{3 t-s}$ for $j=0, \ldots, e-1$ then the extension of $D$ to $R$ will be convergent and will map $R$ into $R$.

Proof. We choose the same symbol $D$ for the extension of the given higher derivation. Application of the defining properties of a higher derivation to $D_{i}(f(\pi))=0$ yields

$$
\begin{aligned}
f^{\prime}(\pi) D_{i}(\pi)= & -f^{D_{i}(\pi)-} \sum_{j_{1}+\cdots+j_{e}=i ; 0 \leqq j_{q}<1} D_{j_{1}}(\pi) \cdots D_{j_{e}}(\pi) \\
& -\sum_{t=0}^{n-1} \sum_{j_{0}+\cdots+j_{t}=i ; 0 \leqq j_{q}<i} D_{j_{0}}\left(f_{t}\right) D_{j_{1}}(\pi) \cdots D_{j_{t}}(\pi)
\end{aligned}
$$

where $f^{D_{1}}=D_{i}\left(f_{e-1}\right) X^{e-1}+\cdots+D_{i}\left(f_{0}\right)$. For $i=1$ we have the familiar formula $D_{1}(\pi)=f^{D_{t}}(\pi) / f^{\prime}(\pi)$ and hence, since $D_{1}\left(f_{j}\right) \in\left[f^{\prime}(\pi)\right]^{2} M^{t-j}$ for $j=0, \ldots, e-1$ we have $D_{1}(\pi) \in f^{\prime}(\pi) M^{t}$. If, for $i<r, D_{i}(\pi) \in f^{\prime}(\pi) M^{t}$ then by $(27) D_{r}(\pi) \in f^{\prime}(\pi) M^{t}$. Thus $D(R) \subset R$. In order to show that $\sum D_{i}(\pi)$ converges we assume that for any integer $s, 1<s<r$, there is an integer $N_{s}>e N_{s-1}$ such that if $i>N_{s}$ then $D_{i}\left(f_{j}\right) \in M^{s t}$ for $j=0, \ldots, n$ and $D_{i}(\pi) \in M^{(s-1) t}$. Then since $D$ converges on $R_{1}$ there is an $N$ such that if $i>N$ then $D_{i}\left(f_{j}\right) \in M^{r t}$ for all $j$. Let $N_{r}$ be the larger of $e N$ and $e N_{r-1}$. It follows then from (27) that for $i>N_{r}, D_{i}(\pi) \in M^{(r-1) t}$ and the lemma is proved. 
If $S$ is a set of representatives in $V$ of a $p$-basis for its residue field $k$ then $V=V^{p^{m}}[S]+p^{m} V$ for any $m>0$. Thus there is a finite subset $S_{1}$ of $S$ such that $f_{j} \in V^{p^{3 t}}\left[S_{1}\right]+p^{3 t} V$. Assuming $S$ to be an infinite set we enumerate a countable subset $\left\{s_{i}\right\}_{i=1}^{\infty}$ of $S-S_{1}$ and we define a higher derivation $D \in H_{c}^{M}(V, R)$ by $D_{i}\left(s_{j}\right)=\delta_{i j}\left[f^{\prime}(\pi)\right]^{2}$, for $i, j>0$, and $D(s)=0$ for $s \in S-\left\{s_{i}\right\}_{i=1}^{\infty}$. By Theorem $4, D$ is in $H_{c}^{M}(V, R)$ and is not in $H_{u}(V, R)$ since $D$ does not converge uniformly on $S$. We extend $D$ to $V\left[X_{1}, \ldots, X_{m}\right]$ and hence, by Proposition 2 , to $R_{1}$ by the requirement $D\left(X_{i}\right)=0$ for $i=1, \ldots, n$, using the same symbol for the extended map. Since $\sum D_{i}\left(X_{j}\right)$ converges for $j=1, \ldots, n, D \in H_{c}^{M}\left(R_{1}, R\right), D \notin H_{u}\left(R_{1}, R\right)$. By construction of $D$ the conditions of Lemma 8 are met and hence $D$ extends to a higher derivation in $H_{c}(R, R)$ which is not in $H_{u}(R, R)$.

The following lemma is needed in order to obtain an analogue to Theorem 5 in case the residue field $R$ has characteristic zero.

Lemma 9. Let $k_{0}, k_{1}$, and $k$ be fields such that $k_{0} \subset k_{1} \subset k$. Let

$$
D \in H_{c}\left(k_{1}, k\left[\left[X_{1}, \ldots, X_{n}\right]\right]\right)
$$

and assume $k_{1}$ separable algebraic over $k_{0}$. If $D$ restricted to $k_{0}$ is uniformly convergent then $D$ is also uniformly convergent. If $D \in H\left(k_{1}, k\left[\left[X_{1}, \ldots, X_{n}\right]\right]\right)$ is convergent ( $M$ convergent) on $k$ then

$$
D \in H_{c}\left(k_{1}, k\left[\left[X_{1}, \ldots, X_{n}\right]\right]\right) \quad\left(D \in H_{c}^{M}\left(k_{1}, k\left[\left[X_{1}, \ldots, X_{n}\right]\right]\right)\right) .
$$

Proof. Let $u$ be in $k_{1}$ and let $f$ be its minimal polynomial over $k_{0}$. If

$$
f=X^{n}+\sum_{i=0}^{n-1} f_{i} X^{i}
$$

then, as in the proof of Lemma 8,

$$
f^{\prime}(u) D_{i}(u)=-f^{D_{i}}(u)-\sum_{j_{1}+\cdots+j_{n}=i ; 0 \leqq j_{t}<i} D_{j_{1}}(u) \cdots D_{j_{n}}(u)
$$

$$
-\sum_{t=0}^{n-1} \sum_{j_{0}+\cdots+j_{t}=i ; 0 \leqq j_{q}<i} D_{j_{0}}\left(f_{t}\right) D_{j_{1}}(u) \cdots D_{j_{t}}(u)
$$

for $i=1,2, \ldots$.

Using (28) and induction on $i$ we observe below that $D_{i}(u)$ is a sum of terms of the form

$$
b D_{i_{1}}\left(a_{1}\right) \cdots D_{i_{r}}\left(a_{r}\right), \quad i_{1}+\cdots+i_{r}=i .
$$

Relation $(28, i)$ exhibits a representation of $D_{(1)}(u)$ as a sum of terms of the form $(29,1)$. Assuming that, for $i<j, D_{i}(u)$ is a sum of the form $(29, \mathrm{i})$ we substitute these sums in $(28, \mathrm{j})$ and conclude that $D_{j}(u)$ is of the same form. The first assertion of Lemma 9 now follows from Lemma 4.

Let $D \in H\left(k_{1}, k\left[\left[X_{1}, \ldots, X_{n}\right]\right]\right)$ be convergent on $k_{0}$ and let $u$ be as above. Now $f^{\prime}(u) D_{i}(u)$ was observed to be a sum of terms of the form $(29, i)$ from which fact 
one concludes that $\sum D_{i}(u)$ converges if $D$ converges on $k_{0}$. The remaining statement is obvious.

THEOREM 6. If $(R, M)$ is a complete regular local ring having residue field $k$ with characteristic zero then $H_{c}(R, R)=H_{u}(R, R)$ if and only if $k$ has finite transcendency degree over its prime field.

Proof. In this case $R$ is a power series ring $k\left[\left[X_{1}, \ldots, X_{n}\right]\right]$ in $n$-indeterminates over $k$. Let $k_{0}$ be the prime field of $k$ and let $B$ be a transcendency basis of $k$. Then, by Proposition 2 and Lemma 9, it is sufficient to show that $H_{c}\left(k_{0}(B), R\right)$ $=H_{u}\left(k_{0}(B), R\right)$ if and only if $B$ is finite. Since the first nonzero mapping of a higher derivation is a derivation and there are no nonzero derivations with domain $k_{0}$ it follows that every higher derivation on $k$ is trivial on $k_{0}$. Hence if $D \in$ $H_{c}\left(k_{0}(B), R\right)$ then $D$ is uniformly convergent on $k_{0}$ and, if $B$ is finite, $D$ is uniformly convergent on $k_{0}[B]$ by Lemma 1 and Corollary 3.1, and hence is uniformly convergent on $k_{0}(B)$ by Corollary 3.2.

If $B$ is infinite we choose a countable subset $\left\{b_{i}\right\}_{i=1}^{\infty}=B^{\prime}$ in $B$ and define a $D \in H_{M}\left(k_{0}(B), R\right)$ by the conditions $D_{i}\left(b_{j}\right)=\delta_{i j} X_{1}$, for $i, j \geqq 1$, and $D_{i}(b)=0$ for $i \geqq 1$ and $b$ in $B, b$ not in $B^{\prime} . D$ is $M$-convergent on $k_{0}$ and on $B$ and hence $D$ is in $H_{c}^{M}\left(k_{0}(B), R\right)$ by Lemma 3. Since $D_{j}\left(b_{j}\right) \notin M^{2}$ for all $j, D$ is not uniformly convergent.

\section{REFERENCES}

1. I. S. Cohen, Structure of complete local rings, Trans. Amer. Math. Soc. 59 (1946), 54-106.

2. N. Heerema, Derivations and embeddings of a field in its power series ring. II, Michigan Math. J. 8 (1961), 129-134.

3. - Derivations on p-adic fields, Trans. Amer. Math. Soc. 102 (1962), 346-351.

4. - Derivations and inertial automorphisms of complete regular local rings, Amer. J. Math. 88 (1966), 33-42.

5. J. Neggers, Derivations on $\bar{p}$-adic fields, Trans. Amer. Math. Soc. 115 (1965), 496-504.

6. H. Hasse and F. K. Schmidt, Noch eine Begründung der Theorie der höheren Differentialquotienten in einen algebraischen Funktionenkorper einer Unbestimmten, J. Reine Angew. Math. 177 (1937), 215-237.

7. E. Wishart, Higher derivations on $\bar{p}$-adic fields, Dissertation, Florida State Univ., Tallahassee, 1965.

FLoRida State University, Tallahassee, Florida 